cancer patients. Most relevant interactions described are paclitaxel with antiepileptics, docetaxel with ketoconazole or cyclophosphamide with benzodiazepines. No clinically relevant interactions were found in our patients. Patients with comorbidities on multiple drug therapy (in addition to the drugs used for cancer treatment) would most benefit from pharmaceutical care.

No conflict of interest.

\section{DGI-061 SAFETY OF TRIPLE TREATMENT IN CHRONIC HEPATITIS C}

doi:10.1136/ejhpharm-2013-000276.327

P Marrero, L Lorente, S Valero, I Gil, E Monte, J Ruiz, J Reig, JL Poveda. Hospital Universitari i Politecnic La Fe, Hospital Pharmacy, Valencia, Spain

Background Efficacy of chronic hepatitis C genotype 1 treatment has been improved with protease inhibitors (PIs) telaprevir and boceprevir. However, triple therapy (PI, peginterferon alfa and ribavirin) has increased the number, type and severity of adverse events.

Purpose To assess the safety of triple therapy in the first 12 weeks of treatment with telaprevir and boceprevir used for chronic hepatitis $\mathrm{C}$ treatment in clinical practise.

Materials and Methods Between March and September 2012, all patients treated with telaprevir and boceprevir receiving medicines in the outpatient pharmaceutical care unit of a tertiary hospital were interviewed. Adverse events were collected in a predefined questionnaire. Anaemia, neutropenia and thrombocytopenia were also included as adverse effects if the patient had been treated for any of them. Interviews were conducted during the medicines dispensing (monthly).

Results Fifty-one patients with triple therapy were interviewed; 34 of them were treated with telaprevir and 17 with boceprevir. All patients had at least one adverse event on any of the visits. Globally, the most frequent adverse events were tiredness (84.3\%), digestive disorders $(70.6 \%)$, dermatological disorders $(64.7 \%)$ and influenzalike syndrome (62.7\%). Patients being treated with telaprevir mainly suffered from tiredness (85.3\%) and dermatological disorders (70.6\%). However, tiredness (82.4\%) and mood disorders $(70.6 \%)$ were the most usual adverse events in patients being treated with boceprevir. The frequencies of other side effects are listed in Table 1. Conclusions Efficacy in the first 12 weeks of triple therapy results in a high frequency of adverse events. Information on possible side effects and how to prevent or treat them is important for patients. Since PIs have only recently come onto the market, it is also important to communicate and record any new adverse events not identified in clinical trials.

No conflict of interest.

\section{DGI-062 SORAFENIB, SUNITINIB AND EVEROLIMUS IN METASTATIC RENAL CELL CARCINOMA: EFFICACY AND SAFETY}

doi:10.1136/ejhpharm-2013-000276.328

I Ormazabal Goicoechea, M Polanco Paz, P Sanmartin Fenollera, M Perez Encinas. Hospital Universitario Fundación Alcorcón, Pharmacy, Madrid, Spain

Background Tyrosine kinase inhibitors (TKIs) and m-TOR inhibitors ( $\mathrm{m}$-TORIs) have demonstrated clinical efficacy in patients with advanced renal cell carcinoma (aRCC).
Abstract DGI-061 Table 1

\begin{tabular}{lll}
\hline Adverse event & Telaprevir (\%) & Boceprevir (\%) \\
\hline Influenza-like illness & 61.8 & 64.7 \\
Tiredness & 85.3 & 82.4 \\
Mood disorders & 32.4 & 70.6 \\
Digestive disorders & 67.7 & 76.5 \\
Dermatological disorders & 70.6 & 52.9 \\
Hair lost & 5.9 & 17.7 \\
Non-productive cough & 8.8 & 29.4 \\
Itchy eyes & 0.0 & 5.9 \\
Oral disorders & 32.4 & 33.3 \\
Haemorrhoids & 64.7 & 0.0 \\
Tachycardia & 2.9 & 23.5 \\
Decreased libido & 2.9 & 11.8 \\
Oedema & 11.8 & 11.8 \\
Anaemia & 55.9 & 47.6 \\
Neutropenia & 17.7 & 11.8 \\
Thrombocytopenia & 14.7 & 5.9 \\
\hline
\end{tabular}

Purpose To describe one centre's experience with the use of TKIs and an oral $\mathrm{m}$-TORI in patients with aRCC.

Materials and Methods Retrospective observational study of patients with aRCC treated with TKIs (sorafenib, sunitinib) and an m-TORI (everolimus) from March 2007-May 2012. Variables: demographics, initial ECOG, line number, duration (TT) and reason for stopping treatment, best response (partial response (PR), stable disease (SD), progression) according to clinical and radiological criteria; progression-free survival (PFS) and overall survival (OS) in weeks (w) and toxicity.

Results Of the 22 patients studied $81.8 \%$ were male with an average age of 65.77 years (SD: 11.76): 5 treated with sorafenib, 13 with sunitinib and 4 with everolimus.

Reasons for discontinuing were: 40\% (2/5), 46.15\% (6/13) and $75 \%$ (3/4) progression/clinical worsening; 40\% (2/5), 15.38\% (2/13) and $25 \%(1 / 4)$ toxicity; and 20\% (1/5), 15.38\% (2/13) and $0 \%$ death, for sorafenib, sunitinib and everolimus respectively. Response rates were (except the 5 patients who stopped too early): sorafenib $100 \%$ SD (2/2); sunitinib 25\% SD (3/12), 58.33\% PR (7/12) and $16.6 \%$ progression (2/12) and everolimus $100 \%$ progression (3/3).

Treatment-related adverse events: sorafenib $60 \%$ asthenia and 40\% rash; sunitinib: $53.85 \%$ rash, $46.15 \%$ diarrhoea and $38.46 \%$ neutropenia, mucositis and asthenia, and everolimus: $75 \%$ hypercholesterolemia, $50 \%$ hypertriglyceridemia and $25 \%$ pneumonitis.

Conclusions In our study, median OS was lower than those obtained in pivotal trials, instead, median PFS was higher, except everolimus. Regarding safety, sorafenib had similar toxicity; sunitinib had higher rates of hand-foot syndrome and everolimus had higher rates of hypercholesterolemia. However, the small number of patients limits our conclusions.

No conflict of interest.

\section{DGI-063 STUDY OF THE USE AND EFFECTIVENESS OF DAPTOMYCIN IN A TERTIARY HOSPITAL}

doi:10.1136/ejhpharm-2013-000276.329

M Bullejos Molina, I Rodriguez Pedrosa, J Nazco Casariego, I Gonzalez Perera, J Gonzalez Garcia. Hospital Universitario de Canarias, Servicio De Farmacia, La Laguna, Spain

Abstract DGI-062 Table 1

\begin{tabular}{|c|c|c|c|c|c|c|c|c|c|c|c|}
\hline & \multicolumn{4}{|c|}{ ECOG } & \multicolumn{4}{|c|}{ No. metastatic sites } & \multicolumn{2}{|c|}{ Line number } & \multirow[t]{2}{*}{ Median TT (w) } \\
\hline & 0 & 1 & 2 & No data & 1 & 2 & 3 & Common sites & 1 & $\geq 2$ & \\
\hline SORAFENIB & & $3 / 5$ & & $2 / 5$ & $2 / 5$ & $3 / 5$ & & $40 \%$ Lung $40 \%$ Bones & $4 / 5$ & $1 / 5$ & 5 (IOR: 2-49.5) \\
\hline EVEROLIMUS & $1 / 4$ & $3 / 4$ & & & $2 / 4$ & $1 / 4$ & $1 / 4$ & $50 \%$ Liver & $0 / 4$ & $4 / 4$ & 10 (IOR: 8-12) \\
\hline
\end{tabular}


Background Daptomycin is an antibiotic only active against Gram-positive bacteria, with rapid bactericidal activity, a concentration-dependent and post-antibiotic effect. Indicated for complicated skin or soft tissue infections in adults (cSSTI), right side endocarditis due to Staphylococcus aureus and S. aureus bacteraemia associated with right-side infective endocarditis.

Purpose To perform a retrospective observational study of the use and effectiveness of daptomycin in our hospital.

Materials and Methods We extracted from the hospital computer system (SAP) prescribing data about daptomycin from January to December 2011. The data collected included age, sex, history number, diagnosis, causative organism, prescriber service, treatment duration and reason for suspension.

Results Were treated 85 patients (69\% male) with an average age of 63.3 years (range 22-86 years). The average duration of treatment was 20.5 days. Prescribers' services were: cardiac surgery/cardiology (27\%), UCI (15\%), haematology (12\%), internal medicine $(12 \%)$, nephrology (12\%) and others (22\%). The diagnoses for which daptomycin was used were: $32 \%$ endocarditis, $32 \%$ cSSTI, $20 \%$ bacteraemia, $11 \%$ osteoarticular infection and $5 \%$ others. Microorganisms identified were: $11 \%$ methicillin-resistant S. aureus (MRSA), $20 \%$ coagulase-negative Staphylococcus, $5 \%$ others and $64 \%$ was empirical treatment. In $36.5 \%$ of prescriptions, daptomycin was used as second-line antibiotic treatment, either because the patient did not respond to previous antibiotic treatment $(32 \%)$ or due to side effects (39\% anaemia with linezolid and $29 \%$ renal damage with vancomycin). The reasons for suspending daptomycin were: $77 \%$ for improvement/patient discharge or who ended treatment or switched to oral treatment, $9 \%$ change in treatment and $14 \%$ deceased.

Conclusions In $84 \%$ of cases the prescription complied with the authorised indications in datasheet. Daptomycin was prescribed first-choice in $63.5 \%$ of treatments. In $64 \%$ of case treatment was empirical without subsequent confirmation of the causative organism. It is necessary to establish a mechanism to decrease the rate of use of this antibiotic in the hospital for frontline empirical treatments.

No conflict of interest.

\section{DGI-064 STUDY OF THE USE OF FERRIC CARBOXYMALTOSE (FC) WITHIN THE SYSTEM FOR PREOPERATIVE OPTIMIZATION OF HAEMOGLOBIN (HB) IN SCHEDULED SURGERY}

doi:10.1136/ejhpharm-2013-000276.330

M Gómez Pérez, MA Arias Moya, M Hernández Segurado, FJ Bécares Martínez, M Bonilla Porras, E Castillo Bazán, MI Panadero Esteban, G Toledano Mayoral, B Rodríguez Vargas, EM Martín Gozalo. Hospital Universitario Fundación Jimenez Diaz, Pharmacy, Madrid, Spain

Background In our hospital there is a protocol for preoperative $\mathrm{Hb}$ optimization with the aim of reducing blood transfusions in patients with anaemia and upcoming surgery.

Purpose To evaluate the use of FC in terms of adherence to protocol and effectiveness.

Materials and Methods Descriptive observational study. The study included patients who had received at least one dose of FC in 2011. We collected from the electronic medical record: age, sex, cause of anaemia, iron administered, $\mathrm{Hb}$ level, iron saturation, transferrin and ferritin before administration of IV iron and surgery. We evaluated adherence to the protocol and analytical results.

Results We studied 47 patients with an age range between 23 and 87 years (median $=62$ ). $78.7 \%$ of the patients met the optimization of $\mathrm{Hb}$ protocol (inclusion criteria: anaemia and upcoming surgery). The average increases in $\mathrm{Hb}$ after a single administration of $500 \mathrm{mg}$ and $1000 \mathrm{mg}$ of $\mathrm{FC}$ were $0.6 \mathrm{~g} / \mathrm{dl}$ and $1 \mathrm{~g} / \mathrm{dl}$ respectively. In the case of patients who had also been given other forms of IV iron before surgery (total average dose of iron administered: $1150 \mathrm{mg}$ ) levels increased by a median of $2.05 \mathrm{~g} / \mathrm{dl}$. Erythropoietin was also administered to $32.43 \%$ of the patients. The mean differences in the rest of the analytical parameters studied before and after administration of iron IV were: serum iron: $40.7 \mu \mathrm{g} / \mathrm{dl}$, \%, iron saturation: $15.8 \%$, transferrin: $-41.8 \mathrm{mg} / \mathrm{dl}$ ferritin: $378.1 \mathrm{ng} / \mathrm{ml}$. The median time between administration and surgery was 6 days.

Conclusions Our results show a fast increase in $\mathrm{Hb}$ in a short time. Restriction of the FC implied making a good selection of patients who may benefit from the higher dose (average increase of $2.05 \mathrm{~g} / \mathrm{dl}$ needs an average dose of $1150 \mathrm{mg}$ iron to be administered) and higher speed of action (median time between administration and surgery: 6 days). Its use would be justified for fast increases in $\mathrm{Hb}$ when, due to the impending surgery, with they would not be obtained in time with other presentations of iron.

Mean differences (for average dose of iron administered: $891.89 \mathrm{mg}$ ) in patients who met the $\mathrm{Hb}$ optimization protocol

Abstract DGI-064 Table 1

\begin{tabular}{llllll}
\hline & Hb & Serum iron & \% iron saturation & Transferrin & Ferritin \\
\hline Mean differences & $1 \mathrm{~g} / \mathrm{dl}$ & $40.7 \mu \mathrm{g} / \mathrm{dl}$ & $15.80 \%$ & $-41.8 \mathrm{mg} / \mathrm{dl}$ & $378.1 \mathrm{ng} / \mathrm{ml}$ \\
\hline
\end{tabular}

No conflict of interest.

\section{DGI-065 STUDY USING FOSCARNET IN HAEMATOLOGICAL PATIENTS}

doi:10.1136/ejhpharm-2013-000276.331

E Garcia-Sanz, M Manso Manrique, E Santiago Prieto, A Torralba Arranz. Universitary Hospital Puerta de Hierro Majadahonda, Pharmacy, Madrid, Spain

Background Cytomegalovirus (CMV) commonly affects bone marrow transplant patients causing significant morbidity and mortality. Foscarnet is a broad-spectrum antiviral agent, active against CMV, but is not the treatment of choice.

Purpose To find out why it was prescribed, to cheque the treatment efficacy and its adverse effects.

Materials and Methods Retrospective study (2011). Data were obtained from patient clinical records and the pharmacy database. We produced a database with information on demographics, underlying disease, indication, treatment duration, dosage, adverse effects and treatment results based on PCR viral load negativization. We also examined whether there had been prior treatment with ganciclovir and the reason for the change, or the reason for not starting treatment with ganciclovir.

Results 12 patients ( 8 male) in the haematology department were treated with foscarnet. Median age was 31 . Underlying diseases: aplastic anaemia (3), lymphocytic leukaemia (4), myeloblastic leukaemia (1), Hodgkin's lymphoma (1), Burkitt's lymphoma (1), T-cell lymphoma (1), myelodysplastic syndrome (1). In 10 cases a bone marrow transplant had been performed. The indication was to treat cytomegalovirus infection except one case in which it was used for suspected infection by herpes virus 6 . In 6 patients ganciclovir was not used first (pancytopenia and problems with engraftment). The other 6 patients had been given ganciclovir and switched due to development of resistance (4) and haematological toxicity (2). Treatment started at low doses and increased as tolerated up to $90 \mathrm{mg} / \mathrm{kg}$

Efficacy: The average length of treatment was 11.4 days. The treatment was effective in 11 patients (91.6\%).

Safety: four patients had no toxicity. We found ulcers on the glans (2), impaired renal function (3) (1 of them requiring dialysis and 1 suspension of treatment), hypomagnesemia which responded to magnesium supplements (2) and 3 gastric discomfort. 